\title{
UMA AVALIAÇÃO DA PRODUÇÃO ACADÊMICA BRASILEIRA RECENTE SOBRE CLUSTERS DE NEGÓCIOS
}

\author{
AN ASSESSMENT OF ACADEMIC PRODUCTION OF RECENT BUSINESS CLUSTERS \\ IN BRAZIL
}

\section{UNA EVALUACIÓN DE LA PRODUCCIÓN ACADÉMICA BRASILEÑa RECIENTE SOBRE CLUSTERS DE NEGOCIOS}

\section{João Paulo Lara de Siqueira}

Doutor em Administração pela Faculdade de Economia, Administração e Contabilidade - FEA/USP

Professor do Programa de Mestrado em Administração da Universidade Paulista - UNIP

E-mail: jp_unip@hotmail.com (Brasil)

\section{Daniel Portillo Serrano}

Mestrando do Programa de Mestrado em Administração da Universidade Paulista - UNIP

Professor de Marketing e Estudos de Comportamento do Consumidor na Faculdade ENIAC

E-mail: danielserrano@uol.com.br (Brasil)

\section{Irene Pereira de Oliveira Stenzel Rimonato}

Mestranda do Programa de Mestrado em Administração da Universidade Paulista - UNIP

Professora da Universidade Nove de Julho - UNINOVE

E-mail: irenepo@ig.com.br (Brasil)

\section{Regina Tartareli}

Mestranda do Programa de Mestrado em Administração da Universidade Paulista - UNIP

E-mail: reginatartareli@ig.com.br (Brasil) 


\section{UMA AVALIAÇÃO DA PRODUÇÃO ACADÊMICA BRASILEIRA RECENTE SOBRE CLUSTERS DE NEGÓCIOS}

\section{RESUMO}

Geralmente os pesquisadores enfrentam dificuldades para identificar os principais autores e textos do tema pelo qual se interessam, principalmente no caso de áreas ainda não muito consolidadas, como é o caso dos clusters de negócios. Este trabalho teve como objetivo identificar os autores nacionais mais importantes de artigos recentes sobre clusters de negócios - em termos de volume de produção e número de citações - e quais os autores estrangeiros que mais influenciam a pesquisa brasileira nessa área. Isso foi feito com uma amostra da literatura de interesse constituída pelos artigos apresentados no Enanpad e no Semead no período de 1997 a 2008 e com a utilização da bibliometria, uma técnica quantitativa e estatística de medição dos índices de produção e disseminação do conhecimento científico. Os resultados indicaram que Toledo, Szafir-Goldstein e Câmara são os autores mais produtivos, que os autores nacionais mais citados são Casarotto Filho, Pires, Amato Neto, Toledo e Zaccarelli e que os autores estrangeiros mais citados são Porter e outros, ligados à área da economia. O estudo sugere que a produção nacional de literatura sobre clusters encontra-se mais dispersa do que a de outras áreas do conhecimento.

Palavras-chave: Clusters; Bibliometria; Estratégia.

\section{AN ASSESSMENT OF ACADEMIC PRODUCTION OF RECENT BUSINESS CLUSTERS IN BRAZIL}

\section{ABSTRACT}

Researchers often face difficulties when identifying the principal texts and authors in a field of knowledge of interest to them, especially in areas that are not consolidated, as in the case of business clusters. This study's objective is to identify the most important authors of recent papers on business clusters, in terms of production volume and number of citations, and which international authors play a major influence on the national research in this area. The study was conceived with a sample of the literature of interest constituted by the works presented in the EnANPAD and Semead events, between 1997 and 2008. The bibliometric technique was adopted a quantitative and statistical method to measure the production and dissemination of scientific knowledge -. Our findings demonstrate that Toledo, Szafir-Goldstein and Câmara are the most published national authors. The researchers having the largest number of citations are Casarotto Filho, Pires, Amato Neto, Toledo and Zaccarelli. International authors possessing a higher number of citations are Michael Porter and others related to the field of economics. The study suggests that the national literature concerned with clusters is more disperse than in other branches of knowledge.

Keywords: Clusters; Bibliometrics; Strategy. 


\section{UNA EVALUACIÓN DE LA PRODUCCIÓN ACADÉMICA BRASILEÑa RECIENTE SOBRE CLUSTERS DE NEGOCIOS}

\section{RESUMEN}

Generalmente los investigadores enfrentan dificultades para identificar los principales autores y textos del tema por el cual se interesan, principalmente en el caso de las áreas que aún no son muy consolidadas, como es el caso de los clusters de negocios. Este trabajo tuvo como objetivo identificar los autores nacionales más importantes de artículos recientes sobre clusters de negocios en términos de volumen de producción y número de citaciones - y cuáles son los autores extranjeros que más influyen en la investigación brasileña en esa área. Esto fue hecho con una muestra de la literatura de interés constituida por los artículos presentados en el Enanpad y en el Semead en el período de 1997 a 2008 y con la utilización de la bibliometría, una técnica cuantitativa y estadística que mide los índices de producción y diseminación del conocimiento científico. Los resultados indicaron que Toledo, Szafir-Goldstein y Câmara son los autores más productivos, que los autores nacionales más citados son Casarotto Filho, Pires, Amato Neto, Toledo y Zaccarelli y que los autores extranjeros más citados son Porter y otros, ligados al área de la economía. El estudio sugiere que la producción nacional de la literatura sobre clusters se encuentra más dispersa que la de las otras áreas del conocimiento.

Palabras-clave: Clusters; Bibliometría; Estrategia. 
Uma Avaliação da Produção Acadêmica Brasileira Recente Sobre Clusters de Negócios.

\section{INTRODUÇÃO}

Ao se dedicar à exploração de um campo de pesquisa, um dos desafios que o pesquisador encontra é o de identificar os autores e as obras mais relevantes, que servirão de base para seus estudos e irão constituir o referencial teórico de sua pesquisa. A seleção de autores e obras é uma etapa sensível, cujas opções irão afetar os resultados do estudo, de forma que essa tarefa, além de árdua, é também de grande responsabilidade. Normalmente a escolha dos autores e textos se dá após uma quantidade razoável de leituras sobre o tema, consultas a especialistas e, até certa medida, por meio de um processo intuitivo, uma vez que raramente o pesquisador tem acesso a todos os textos publicados e, de antemão, possui a perícia necessária para saber quais obras são as mais relevantes.

Quando a pesquisa se dá em um campo emergente do conhecimento, como o do estudo dos clusters de negócios, as dificuldades são ainda maiores, pois a produção científica é esparsa, o conhecimento apresenta um baixo grau de estruturação e, em geral, os principais autores ainda não estão consagrados como tal.

Uma técnica que vem sendo empregada em diversas áreas, inclusive a administração, com a finalidade de mapear a produção científica, identificando os principais autores, as obras (artigos, livros ou textos) e revistas mais influentes é a bibliometria. São exemplos da aplicação da bibliometria na área de gestão o trabalho de Acedo et al. (2006), que investigaram a disseminação e as principais tendências da Resource-Based Theory - RBT nos periódicos mais importantes de administração e o de Dombrow e Turbull (2002), sobre os indivíduos e instituições que publicaram pesquisas acerca do mercado imobiliário entre os anos de 1989 e 1998.

A técnica bibliométrica também tem sido utilizada no Brasil, como atestam os estudos de Lunardi et al. (2005) sobre as publicações de sistemas de informação no ENANPAD e nas principais revistas brasileiras de Administração (classificadas pela CAPES como “A”), de Benetti et al. (2008), que avaliou o estado da arte dos artigos de estratégia publicados na Revista de Administração Contemporânea entre 2005 e 2007 e de Moretti e Campanário (2009), que abordou as publicações brasileiras na área da Responsabilidade Social Empresarial por meio da análise dos trabalhos apresentados no EnANPAD, entre 1997 e 2007.

Segundo Araújo (2006), a bibliometria também permite, por meio da análise das citações encontradas nas publicações, identificar:

"autores mais citados, autores mais produtivos, elite de pesquisa, frente de pesquisa, fator de impacto dos autores, procedência geográfica e/ou institucional dos autores mais influentes em um determinado campo de pesquisa; tipo de documento mais utilizado, idade

Revista Ibero-Americana de Estratégia - RIAE, São Paulo, v. 10, n. 1, p. 55-76, jan/abr. 2011. 
média da literatura utilizada, obsolescência da literatura utilizada, procedência geográfica e/ou institucional da bibliografia utilizada; periódicos mais citados, "core" de periódicos que compõem o campo.”.

Em um estudo sobre a gestão da informação e do conhecimento, Guedes e Borschiver (2005), mencionam alguns princípios e leis da bibliometria, destacando como principais a lei de Bradford sobre a produtividade de periódicos, a lei de Zipf sobre a frequência de palavras em textos científicos e a lei de Lotka sobre a produtividade dos autores. O padrão das leis e princípios bibliométricos, segundo Guedes e Borschiver (2005), seria o da máxima "poucos com muito e muito com poucos". Por exemplo, no caso específico da lei de Lotka, a premissa básica seria a de que "alguns pesquisadores publicam muito e muitos publicam pouco" (VOOS, 1974, apud GUEDES e BORSCHIVER, 2005). Esse fato foi corroborado por Price, que, ao realizar um trabalho que visava o aperfeiçoamento da lei de Lotka, e "a partir de estudos realizados entre 1965 e 1971 , concluiu que $1 / 3$ da literatura é produzida por menos de $1 / 10$ dos autores mais produtivos" (ARAÚJO, 2006).

Posto isso, fica clara a importância da identificação desse grupo de pesquisadores relativamente pequeno que mais contribui para o avanço do conhecimento em determinada área. Neste artigo é apresentada uma aplicação de algumas técnicas da bibliometria para exploração da produção brasileira sobre o tema clusters de negócios, com foco nos seus principais autores e nas influências que estes receberam e que mais contribuíram para seus trabalhos.

\subsection{PROBLEMA DE PESQUISA E OBJETIVO}

Como já foi mencionado, os estudiosos dos clusters de negócios, com maior ou menor intensidade, costumam enfrentar dificuldades para selecionar textos e autores para embasar seus estudos. Essa dificuldade é comum em áreas em que o conhecimento ainda não se encontra consolidado ou há atividade de investigação intensa e, consequentemente, manter-se atualizado é uma tarefa mais complexa.

A análise dos autores que mais publicam sobre um tema e dos que recebem maior quantidade de citações é uma forma de medir a importância ou a produção dos pesquisadores (ARAÚJO, 2006; GUEDES e BORSCHIVER, 2005; PAULA et al., 2010; MORETTI e CAMPANÁRIO, 2009 entre outros). O problema de pesquisa que se coloca é a identificação dos pesquisadores-autores que mais têm influenciado os estudiosos brasileiros de clusters e que mais contribuições dão para a compreensão desse fenômeno.

Revista Ibero-Americana de Estratégia - RIAE, São Paulo, v. 10, n. 1, p. 55-76, jan/abr. 2011. 
Uma Avaliação da Produção Acadêmica Brasileira Recente Sobre Clusters de Negócios.

Dessa forma, a questão de pesquisa que se coloca é: quais são os autores nacionais mais importantes na área de clusters de negócios e quais são os autores internacionais que mais os influenciam? Além disso, pretende-se verificar se na produção nacional sobre clusters de negócios podem ser observados o percentual de $1 / 3$ da literatura sendo produzido por menos de 1/10 dos autores mais produtivos, conforme apontado genericamente por Price.

Assim sendo, o objetivo geral deste artigo é identificar os autores nacionais de artigos recentes sobre clusters de negócios mais importantes - em termos de volume de produção e número de citações por autores - e quais os autores estrangeiros que mais influenciam a pesquisa brasileira na área de clusters, além de analisar a dispersão dessa produção.

Constituem objetivos específicos:

1. Identificar os autores nacionais mais produtivos;

2. Identificar os autores nacionais mais citados por autores nacionais (isto é, que têm maior impacto sobre aqueles que pesquisam clusters);

3. Identificar os autores estrangeiros mais citados pelos autores nacionais (e seu respectivo impacto);

4. Verificar se na produção nacional sobre clusters é observada uma dispersão de acordo com a Lei de Lotka, conforme enunciada por Price.

Este artigo está estruturado em cinco partes, iniciando-se pela introdução, a apresentação do problema de pesquisa e havendo, a seguir, uma revisão preliminar da literatura, a apresentação da metodologia, a análise dos resultados obtidos e as conclusões.

Com relação à revisão da literatura, é importante destacar seu caráter preliminar, isto é, não foram considerados os resultados obtidos na pesquisa bibliométrica, que foi realizada em uma etapa posterior dos trabalhos. Os autores e textos utilizados na revisão bibliográfica foram selecionados por meio de consultas feitas pelos autores à especialistas no assunto, representando, portanto, um ponto de vista sobre o tema.

Revista Ibero-Americana de Estratégia - RIAE, São Paulo, v. 10, n. 1, p. 55-76, jan/abr. 2011. 


\section{REVISÃO BIBLIOGRÁFICA}

A revisão da literatura abrange textos sobre clusters de negócios indicados por especialistas e também sobre conceitos relativos à técnica da bibliometria. Aspectos mais operacionais sobre bibliometria também são abordados na metodologia.

\subsection{BIBLIOMETRIA}

A bibliometria é vista como uma "técnica quantitativa e estatística de medição dos índices de produção e disseminação do conhecimento científico" (ARAÚJO, 2006), "um conjunto de leis e princípios empíricos que contribuem para estabelecer os fundamentos teóricos da Ciência da Informação" (GUEDES e BORSCHIVER, 2005) ou, ainda, "uma análise quantitativa de variáveis do discurso" (LIMA, 1984).

Pritchard (1969) é apontado por autores mais recentes (GUEDES e BORSCHIVER, 2005; MORETTI e CAMPANÁRIO, 2009) como quem pela primeira vez usou o termo bibliometria. Para ele, bibliometria significa "todos os estudos que tentam quantificar os processos de comunicação escrita".

O objeto da bibliometria é a análise da produção científica e de seus conteúdos. Isso é feito por meio da quantificação da produção e com o uso de processos variados de levantamento, tratamento e apresentação dos dados. Essa abordagem permite a construção de mapas do conhecimento, que incluem inter-relacionamentos e produtividade (MORETTI e CAMPANÁRIO, 2009).

Ramos-Rodríguez e Ruíz-Navarro (2004), citados por Acedo et al. (2006), destacam como uma das vantagens da bibliometria ser um método objetivo, capaz de examinar a evolução e as tendências de um campo de estudo, sem que isso seja baseado na opinião de especialistas, que, por serem subjetivas, podem conter vieses.

O emprego das técnicas de bibliometria é justificado pela necessidade dos pesquisadores de terem mapeadas as áreas do conhecimento pelas quais se interessam, pelo compromisso que os bibliotecários têm de manter seus acervos atualizados em relação às obras relevantes e também, como lembra Strehl (2005) pelo fato de que "a crescente demanda por financiamento de atividades científicas tem tornado necessário o estabelecimento de critérios mais exigentes do que aqueles até então utilizados na avaliação de pesquisadores e instituições”.

Atualmente a bibliometria é aplicada nas mais diversas áreas do conhecimento. Van Leeuwen (2005), em um artigo sobre a aplicação da bibliometria na avaliação das ciências sociais, 
sugere que o argumento de que nessa área a bibliometria não poderia ser usada porque o padrão de citações é muito diferente do que é encontrado nas ciências naturais e da vida, deve ser contornado com a análise de um maior número de publicações e uma janela de tempo maior. Nesse seu artigo, especificamente, foi considerado um intervalo de tempo de 10 anos. $\mathrm{O}$ autor, inclusive, afirma que “estudos, por exemplo, no Reino Unido e no Canadá, têm indicado as possibilidades e vantagens da aplicação da bibliometria na avaliação das ciências sociais” (VAN LEEUWEN, 2005).

Uma das análises possíveis nos estudos bibliométricos é a análise de citações. Segundo Acedo et al. (2006), "as citações de um artigo científico indicam os fundamentos teóricos e empíricos do estudo e a análise das citações torna possível identificar redes de autores e de textos pertencentes às mesmas escolas, paradigmas ou teorias”. A análise de citações mostra o que foi publicado em determinado corte da literatura e relaciona o citante com o citado, dirigindo o leitor para outras fontes de informações correlatas (LIMA, 1984).

Os estudos bibliográficos podem ser utilizados para o conhecimento do fator de impacto de uma publicação. Segundo Bergstrom e West (2008) e também Strehl (2005), o fator de impacto é a medida do número médio de citações que os artigos de um periódico recebem ao longo dos dois anos seguintes à sua publicação. De forma semelhante, o Journal Citation Reports define fator de impacto como "a medida da frequência com a qual o artigo médio de um periódico foi citado em um particular ano ou período".

Entre as leis e princípios existentes no campo da bibliometria encontra-se a lei de Lotka, relativa à produtividade dos pesquisadores e segundo a qual se deve esperar que exista uma concentração da produção científica em alguns poucos autores. A lei de Lotka é "considerado o eixo central da pesquisa bibliométrica contemporânea” (MORETTI; CAMPANÁRIO, 2009).

De acordo com a lei de Lotka, o número de autores que fazem $n$ contribuições em um determinado campo científico é aproximadamente $1 / \mathrm{n}^{2}$ daqueles que fazem apenas uma contribuição e a proporção daqueles que fazem uma única contribuição é de mais ou menos $60 \%$ (URBIZAGÁSTEGUI ALVARADO, 2002).

Entre os aperfeiçoamentos que a lei de Lotka recebeu ao longo dos anos, desde sua formulação original em 1926, destaca-se o trabalho de Price, que concluiu que 1/3 da literatura é produzida por menos de 1/10 dos autores mais produtivos, levando a uma média de 3,5 documentos por autor e $60 \%$ dos autores produzindo um único documento (Araújo, 2006).

Em uma revisão da literatura sobre a aplicação da lei de Lotka à produção científica brasileira em diversas áreas, Urbizagástegui Alvarado (2002) encontrou resultados contraditórios que vão desde a não conformidade dos dados à Lei a desvios consideravelmente altos, dados que se

Revista Ibero-Americana de Estratégia - RIAE, São Paulo, v. 10, n. 1, p. 55-76, jan/abr. 2011. 
ajustam mais claramente à formulação proposta por Price e até um adequado ajuste à Lei de Lotka propriamente dita. A tabela 1 apresenta esses resultados.

Tabela 1 - Aplicações brasileiras da lei de lotka.

\begin{tabular}{|c|c|c|c|c|c|}
\hline Área & $\begin{array}{l}\text { Período } \\
\text { estudado }\end{array}$ & $\begin{array}{l}\text { Cálculo } \\
\text { de C }\end{array}$ & $\begin{array}{l}\text { Teste } \\
\text { de ajuste }\end{array}$ & $\begin{array}{l}\text { Forma de } \\
\text { contagem }\end{array}$ & $\begin{array}{l}\text { Ajuste à Lei } \\
\text { de Lotka }\end{array}$ \\
\hline Direito & 5 anos & $1 / n^{2}$ & nenhum & não indica & $\operatorname{sim}$ \\
\hline Medicina & 2 anos & $1 / n^{2}$ & nenhum & não indica & não indica \\
\hline Microbiologia & 1 ano & $1 / n^{2}$ & nenhum & não indica & $\operatorname{sim}$ \\
\hline Arquivo & 10 anos & $1 / n^{2}$ & nenhum & não indica & não \\
\hline Siderurgia & 13 anos & $1 / n^{3}$ & nenhum & não indica & não \\
\hline Veterinária & 5 anos & $1 / n^{2}$ & nenhum & direta & não \\
\hline Saúde Publica & 6 anos & $1 / n^{2}$ & nenhum & não indica & $\operatorname{sim}$ \\
\hline Jaca & 31 anos & $1 / \mathrm{n}^{2}$ & nenhum & não indica & não \\
\hline Biblioteconomia & 6 anos & $1 / n^{2}$ & nenhum & não indica & $\operatorname{sim}$ \\
\hline Universidade & 2 anos & $1 / n^{2}$ & nenhum & direta & $\operatorname{sim}$ \\
\hline Marketing & 23 anos & $1 / n^{2}$ & nenhum & não indica & $\operatorname{sim}$ \\
\hline
\end{tabular}

Fonte: URBIZAGÁSTEGUI ALVARADO, R. A Lei de Lotka na bibliometria brasileira Ci. Inf., Brasília, V. 31, N. 2, 2002, pp. 14-20.

\subsection{CLUSTERS DE NEGÓCIOS}

Há algum tempo, o fenômeno dos clusters de negócios vem despertando o interesse dos pesquisadores, gerando um número razoável de publicações em periódicos de prestígio de editoras consagradas. Trata-se de um tema multidisciplinar, que abrange as áreas da economia, da administração, da geografia econômica, da ciência política e da sociologia, entre outras. A identificação da capacidade dos clusters de negócios para estimular o desenvolvimento da economia de uma região ou país tem levado políticos, agências de desenvolvimento e governos a se interessar por eles, reconhecendo-os como uma opção para estimular e ampliar a competitividade de indústrias, de reduzir desigualdades entre regiões ou de criar polos de desenvolvimento.

A literatura sobre os clusters, inicialmente, centrou-se nas concentrações de manufaturas, sendo, provavelmente, seu maior exemplo, os trabalhos de Porter (1990), envolvendo a produção de revestimentos cerâmicos na Itália, instrumentos de monitoramento médico nos EUA, máquinas de impressão na Alemanha e robôs no Japão. Posteriormente foram publicados trabalhos sobre clusters turísticos e alguns poucos sobre clusters comerciais.

O primeiro passo no sentido de oferecer uma teorização sobre a configuração de negócios, que mais tarde viria a ser chamado genericamente de cluster, foi dado pelo economista inglês Alfred Marshall (1982), que, ao final do Século XIX, observou a existência de artigos que eram 
Uma Avaliação da Produção Acadêmica Brasileira Recente Sobre Clusters de Negócios.

"produzidos em alguns lugares apenas, ou mesmo num único lugar, e se difundiam por toda a Europa". Segundo esse autor, tais artigos seriam provenientes de uma concentração de indústrias em certas localidades e esse fenômeno seria chamado comumente, "embora não muito acertadamente", de "indústria localizada" (MARSHALL, 1982, p.231). Além de identificar a concentração de indústrias, Marshall também verificou algumas vantagens ligadas a essas configurações produtivas: disponibilidade de trabalhadores com mais aptidão - pois dentro das concentrações "os segredos da profissão deixam de ser segredos" (p. 234) - e com mais capacidade de inovar, o surgimento no local de atividades subsidiárias e a possibilidade de ratear os custos para incorporação de novas tecnologias (então mencionadas como "maquinário especializado").

Desde então, diferentes pesquisadores se interessaram pelos agrupamentos regionais de empresas, como Krugman (1991), que, em seus trabalhos sobre geografia econômica, relatou o caso do cluster de colchas para camas e tapetes em Dalton, na Geórgia (EUA), exemplificando como um evento fortuito pode se constituir no ponto de partida para o desenvolvimento de um cluster e mostrando como a teoria econômica pode ser empregada para explicar porque negócios semelhantes são atraídos para um local, uma vez iniciado o processo de agrupamento de atividades afins.

Embora não exista um conceito de cluster universalmente aceito, provavelmente a concepção de Porter seja a mais conhecida. Em artigo publicado em 1998, Porter definiu "concentrações geográficas de companhias e instituições interconectadas em um campo particular" como sendo um cluster, citando como exemplos as vinícolas da Califórnia, o Vale do Silício, a indústria automobilística em Detroit, os cassinos em Las Vegas e os serviços financeiros em Nova York. Na Europa, Porter identificou os clusters de moda de couro na Itália e da indústria química na Alemanha.

Anteriormente, Porter (1990) já havia apresentado os clusters da indústria robótica japonesa, de azulejos de cerâmica em Sassuolo, na Itália, de máquinas impressoras na Alemanha e de equipamentos de monitoração de pacientes nos EUA.

Altenburg e Meyer-Stamer (1999) afirmam que "no seu sentido mais amplo, o termo cluster apenas descreve concentrações locais de certas atividades econômicas" e que seu interesse reside no fato de apresentarem uma "eficiência coletiva", que não seria atingida por aglomerações de firmas não relacionadas. Essa eficiência coletiva teria origem em externalidades positivas, custos de transação baixos e ação conjunta.

A definição operacional adotada no estudo desses últimos autores é a de que "um cluster é uma aglomeração considerável de firmas em uma área espacialmente delimitada na qual há um perfil diferenciado de especialização e existem negócios e especialização entre empresas de forma 
substancial".

Perry (2005), embora concorde com a interpretação frequente de que clusters estejam associados à "concentração de uma atividade em uma localidade específica", ressalta que essa concepção carece de elaboração, pois "não permite distinguir um cluster de formas inferiores de agrupamento" (PERRY, 2005, p.11).

Esse autor pondera que existem várias perspectivas para o uso do termo cluster e se identifica mais com duas delas: a primeira, que compreende os clusters como uma condição de localização particular, segundo a qual clusters seriam um "agrupamento geográfico industrial distinto que possui a capacidade de obter vantagem sobre agrupamentos de atividade econômica alternativos" (PERRY, 2005, p.12), e a segunda, para a qual os clusters são economias de alto desempenho, isto é,

localidades onde companhias estão presas, conjuntamente, por várias formas de interdependência, como organismos em uma biosfera. Os negócios competem entre si por participação de mercado, empregados e recursos, até mesmo mais vigorosamente do que aqueles que estão fora do cluster. Ao mesmo tempo, os negócios dependem uns dos outros (PERRY, 2005, p.12).

No Brasil, como em outros países interessados em formas de estimular o desenvolvimento regional, os clusters têm despertado a atenção de diversos pesquisadores. Segundo Lastres e Cassiolato (2003), "o termo cluster associa-se à tradição anglo-americana e, genericamente, referese a aglomerados territoriais de empresas, desenvolvendo atividades similares". Zaccarelli (2004, p. 197), utilizando-se do ponto de vista estratégico, afirma que "cluster significa um agrupamento de objetos similares", devendo ser entendido como um agrupamento competitivo e que "o distrito industrial de uma cidade formará um cluster se todas as empresas aí presentes se dedicarem a produzir o mesmo tipo de produto e se o agrupamento dessas empresas fizer com que a competição assuma características diferentes.”.

$\mathrm{Na}$ literatura da área, são encontrados textos sobre clusters e também sobre os chamados arranjos produtivos locais - APL. Em alguns textos, clusters e APL's são usados quase como sinônimos, ao passo que em outros parece haver algum tipo de diferenciação entre eles.

Para Lastres e Cassiolato (2003), arranjos produtivos locais são aglomerações territoriais de agentes econômicos, políticos e sociais focados em atividades econômicas específicas e que apresentam vínculos entre si. Geralmente envolvem empresas produtoras de bens e serviços finais e seus fornecedores, comercializadoras, clientes, associações e organizações voltadas para pesquisa, desenvolvimento, política, promoção, financiamento e também a formação e capacitação de recursos humanos, como escolas técnicas e universidades.

Revista Ibero-Americana de Estratégia - RIAE, São Paulo, v. 10, n. 1, p. 55-76, jan/abr. 2011. 
Uma Avaliação da Produção Acadêmica Brasileira Recente Sobre Clusters de Negócios.

Em uma perspectiva próxima, mas de certa forma mais focalizada na operação do que na configuração desses arranjos, Suzigan et al. (2003) afirmam esses sistemas produtivos locais podem possuir empresas de portes variados, mas geralmente têm um conjunto expressivo de pequenas e médias empresas não integradas verticalmente. Esses autores também apontam a presença de importantes instituições locais de apoio às empresas.

A comparação entre o posicionamento desses autores em relação a clusters e APL's indica diferença discreta entre conceitos, podendo-se identificar, como aspecto comum, a consideração de aglomerações territoriais de empresas semelhantes e afins e, como aspecto divergente, a presença nos APL's de uma conotação mais voltada ao desenvolvimento, em função da preconização de instituições de apoio, como escolas técnicas e universidades; pesquisa, desenvolvimento e engenharia; política, promoção e financiamento.

A observação e a análise de clusters de negócios, sob a ótica da estratégia, sugerem que esse tipo de agrupamento manifesta uma natureza sistêmica, cujo reconhecimento conduz a uma compreensão mais ampla do próprio fenômeno cluster. Nesse sentido, Zaccarelli et al. (2008) afirmam que o conjunto de empresas de um cluster forma um sistema, que pode ser entendido como uma entidade supra-empresarial, e que, embora essa entidade possa ser considerada "uma abstração" (p. 43), ela apresenta comportamento com características próprias, que não existem nas empresas isoladamente. Esses pesquisadores definem entidade supraempresarial como um sistema instituído pelo relacionamento entre um conjunto de negócios ligados a um produto, linha, categoria ou mercado, que apresenta efeitos sistêmicos de amplificação da capacidade competitiva do sistema e de seus componentes em relação a empresas situadas externas a ele.

\section{METODOLOGIA}

Entende-se método como "o conjunto das atividades sistemáticas e racionais que, com maior segurança e economia, permite alcançar o objetivo" (MARCONI; LAKATOS, 2009). Tendo em vista os objetivos definidos para o presente estudo, decidiu-se pelo emprego do método indutivo, com a análise dos artigos publicados dentro de um período de tempo específico, em dois eventos que estão entre os principais na área de administração no Brasil.

Tal procedimento é semelhante ao empregado por Pegino (2005), que, ao pesquisar as bases filosóficas das publicações em estratégia, selecionou como fonte os textos da Enanpad, especificamente aqueles alocados na área temática "Estratégia em Organizações", fixando-se no 
período entre 2000 e 2004. Nesta investigação foram pesquisados os textos dos anais dos encontros da Anpad de 1997 a 2008, nas áreas de estratégia e administração geral e também os textos das mesmas áreas nas edições do Semead de 2000 a 2008, exceto o de 2007, cujos artigos não se encontravam disponíveis na época da pesquisa.

Uma decisão importante que deve ser tomada pelo pesquisador quando da realização de uma pesquisa bibliométrica é quanto ao foco do estudo, que pode ser em artigos (textos), periódicos, autores ou combinações desses elementos. Neste estudo, em função do objetivo estipulado, optou-se pelo foco em autores.

No presente artigo, especialistas consultados pelos pesquisadores sugeriram 11 autores como prováveis fontes de citação e a frequência com que esses autores foram citados nos textos analisados foi contabilizada. Além desses nomes, selecionados de antemão, complementarmente também foram verificados todos os nomes constantes das referências, de forma que eventuais falhas na listagem de autores provavelmente mais citados pudessem ser corrigidas.

Os 11 autores selecionados a priori são os que se encontram relacionados na tabela 2, a seguir:

Tabela 2 - Autores selecionados a priori.

\begin{tabular}{|l|}
\hline \multicolumn{1}{|c|}{ AUTOR } \\
\hline PORTER, MICHAEL E. \\
\hline MARSHALL, ALFRED \\
\hline MEYER-STAMER, JÖRG \\
\hline KRUGMAN, PAUL \\
\hline SCHIMITZ, HUBERT \\
\hline ZACCARELLI, SERGIO B. \\
\hline ROSENFELD, STUART A. \\
\hline SUZIGAN, WILSON \\
\hline ALTENBURG, TILMAN \\
\hline LASTRES, HELENA M. M. \\
\hline CASIOLATTO, JOSÉ E. \\
\hline
\end{tabular}

Os dados dos artigos foram coletados com o auxílio de um formulário de observação desenvolvido especificamente para esse fim. De acordo com Boyd e Westfall (1979), deve-se entender por formulário uma lista das informações a serem obtidas pelo pesquisador. Essa lista tem por objetivo simplificar a identificação e o registro exato das informações e facilitar a tabulação dos dados. Informações sobre os autores foram obtidas no sítio do sistema Lattes, do CNPQ.

Como a identificação dos autores mais importantes está ligada diretamente ao problema de pesquisa, buscou-se uma forma de avaliar o impacto desses pesquisadores nas publicações nacionais de clusters. Isso foi feito de forma semelhante ao cálculo do impacto de um periódico. A 
Uma Avaliação da Produção Acadêmica Brasileira Recente Sobre Clusters de Negócios.

avaliação do impacto de um autor foi calculada pela divisão do número total de artigos nos quais ele é citado pelo número total de artigos analisados.

Trata-se, portanto, de uma avaliação interna do impacto, isto é, restrita ao conjunto de artigos analisados. A opção pela avaliação interna do impacto deve-se à análise preliminar dos autores dos artigos estudados. Essa análise mostrou que muitos pesquisadores produzem também em outras áreas da Administração, e caso fosse simplesmente adotada uma média dos fatores de impacto de suas publicações, haveria viés em relação aos quais deles são os mais importantes para os clusters.

Dados os objetivos da pesquisa, a população de interesse do estudo é composta por todos os textos publicados sobre o assunto clusters ou sobre arranjos produtivos locais (conhecidos como APL), que possuam caráter científico e que tenham sido publicados dentro do intervalo de tempo de 1997 a 2008. Dadas as dificuldades já bastante exploradas pela literatura de pesquisa científica de se conduzir estudos censitários, optou-se pela utilização de uma amostra, composta pelos artigos do Enanpad e Semead, conforme já explicitado. Está implícito na amostragem empregada que foi considerado que a produção nacional sobre clusters está representada pelos trabalhos publicados nos anais do Enanpad e Semead e que o foi entendido como recente.

A seleção dos textos a serem pesquisados seguiu o critério usado por Balestrin et al. (2010) e por Oliver e Ebers (1998) que verificaram nos títulos, nos resumos e nas palavras-chave dos artigos termos que indicassem relação com o estudo. Neste caso, os termos procurados foram cluster, aglomerado, aglomeração e arranjo produtivo local - APL. Posteriormente foram excluídos os artigos que apenas utilizavam a técnica estatística de análise de cluster e não pertenciam ao escopo da pesquisa.

As limitações do estudo dizem respeito, em primeiro lugar, à validade interna dos resultados, isto é, à utilização de um processo de amostragem não probabilístico, que inviabiliza o emprego de técnicas estatísticas para generalização dos resultados e, em segundo lugar, a escolha do Enanpad e do Semead para compor amostra, posto que poderiam ter sido escolhidas outras fontes, como as revistas RAUSP, RAE ou RAC - aliás, o que se pretende fazer como prosseguimento da presente investigação.

Revista Ibero-Americana de Estratégia - RIAE, São Paulo, v. 10, n. 1, p. 55-76, jan/abr. 2011. 


\section{ANÁLISE DOS RESULTADOS}

Foram localizados 26 artigos relativos a clusters de negócios nas áreas de estratégia e administração geral nos anais dos encontros da Anpad de 1997 a 2008 e do Semead de 2000 a 2008, exceto 2007. A apresentação dos resultados seguiu o critério usado por Paula et al. (2010), que usaram gráficos (que reúnem as publicações por período e mostram sua evolução com o tempo) e tabelas.

No gráfico 1 é possível observar a quantidade de artigos apresentados em cada ano e verificar que 2005, foi o ano que contou com a maior produção (oito textos), seguido por 2006 (seis textos).

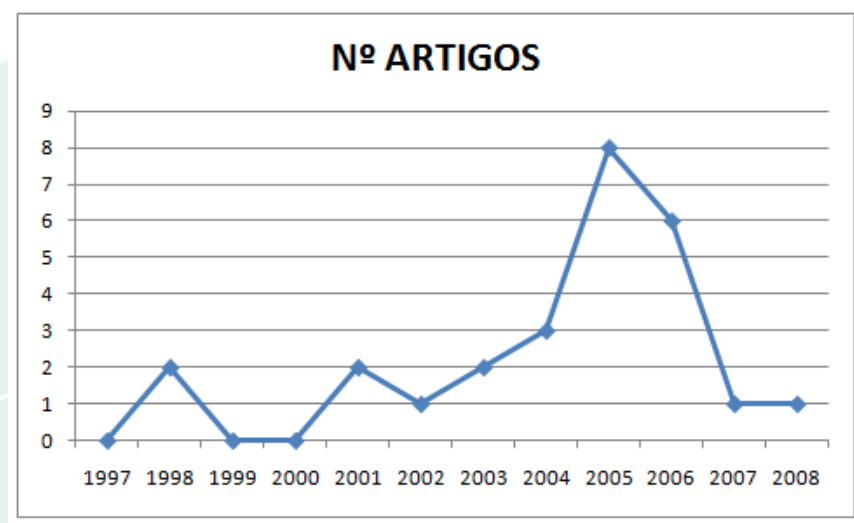

Gráfico 1 - Número de artigos publicados no Enanpad e Semead

Foram identificados todos os autores dos 26 artigos e seus nomes estão apresentados nas tabelas a seguir. Na tabela 2 estão os autores que publicaram apenas um artigo. Observa-se que 59 pesquisadores tiveram um artigo apresentado no Enanpad ou no Semead, no período considerado, e três pesquisadores tiveram mais de um artigo aceito.

Na tabela 3 estão os autores que publicaram mais de um artigo e a quantidade (a frequência) de artigos publicados. O percentual da produção correspondente aos autores que publicaram apenas uma vez foi de $3,486 \%$. O percentual da produção relativo aos autores com mais de uma publicação se encontra na tabela 4.

Revista Ibero-Americana de Estratégia - RIAE, São Paulo, v. 10, n. 1, p. 55-76, jan/abr. 2011. 
Uma Avaliação da Produção Acadêmica Brasileira Recente Sobre Clusters de Negócios.

Tabela 3 - Autores que publicaram UM artigo.

\begin{tabular}{|l|l|}
\hline ALESSANDRA COSTENARO & JOSÉ DE PAULA BARROS NETO \\
\hline ALEXANDRE TEIXEIRA DIAS & JOSE HAMILTON MATURANO CIPOLLA \\
\hline ALMIRALVA FERRAZ GOMES & JUNIA CERCEAU \\
\hline ALVARO CASTROMAN POLERO & LEANDRO CABRAL DE ALMEIDA \\
\hline AMELIA SILVEIRA & LENISE SARAIVA DE VASCONCELOS COSTA \\
\hline ANA AUGUSTA FERREIRA DE FREITAS & LIGIA DUARTE GUERRA \\
\hline ANTONIO TEODORO RIBEIRO MAGALHÃES & LUÍS GUSTAVO MAZZARO \\
\hline BELMIRO DO NASCIMENTO JOÃO & LUIZ ANTONIO ALIGLERI \\
\hline CLAUDIA LUNKES SCHMIT & LUIZCARLOS DOS SANTOS \\
\hline DOUGLAS WEGNER & LUIZ GUSTAVO ANTONIO DE SOUZA \\
\hline EDUARDO MADEIRA MARQUES & MANUEL PORTUGAL FERREIRA \\
\hline FÁBIO LOTTI OLIVA & MARCELO BRONZO LADEIRA \\
\hline FELIPE FONTES RODRIGUES & MARCELO DO CARMO \\
\hline FELIPE MENDES BORINI & MÁRCIA ZABDIELE MOREIRA \\
\hline FERNANDO A. RIBEIRO SERRA, & MARCO AURÉLIO ARBEX \\
\hline FERNANDO ANTONIO PRADO GIMENEZ & MARIA EUGÊNIA MONTEIRO CASTANHEIRA \\
\hline FERNANDO COUTINHO GARCIA & MARIANNE HOELTGEBAUM \\
\hline FERNANDO GOMES DE PAIVA JR & MARLY CAVALCANTI \\
\hline FERREIRA DASILVA & MAURO CALIXTA TAVARES \\
\hline FRANCISCO VIDAL BARBOSA & MAYARA MARIA DE JESUS MUNIZ \\
\hline GILNEI LUIZ DE MOURA & MILTON LUIZ WITTMANN \\
\hline GLAISON AUGUSTO GUERREIRO & MOACIR DE MIRANDA OLIVEIRA.JUNIOR \\
\hline GRAZIELE BEILER & PEDRO PAULO HUGO WILHELM \\
\hline HÉBER JOSÉ DE MOURA & RINALDO MACHADO DE ALMEIDA \\
\hline HÉLIO CÂNDIDO FERREIRA & ROGÉRIO CERÁVOLO CALIA \\
\hline IVAN DE SOUZA DUTRA & RUY BONATES DE ALMEIDA JUNIOR \\
\hline JESÚS ÁLVAREZ VALDÉS & SAMUEL CARVALHO DE BENEDICTO \\
\hline JOÃO CARLOS NEVES DE PAIVA & SANDRA LILIAN DE OLIVEIRA FAÇANHA \\
\hline JOAQUIM BRASILEIRO NETTO & SÉRGIO HENRIQUE ARRUDA CAVALCANTE FORTE \\
\hline & SILVIO ROBERTO STEFANO \\
\hline & \\
\hline
\end{tabular}

Tabela 4 - Autores que publicaram MAIS DE UM artigo.

\begin{tabular}{|l|c|c|}
\hline AUTOR & FREQÜÊNCIA & $\begin{array}{c}\text { PERCENTUAL } \\
\text { DA } \\
\text { PRODUÇÃO }\end{array}$ \\
\hline TOLEDO, GERALDO L. & 4 & $15,385 \%$ \\
\hline SZAFIR-GOLDSTEIN, CLÁUDIA & 3 & $11,538 \%$ \\
\hline CAMARA, MÁRCIA R. G. & 2 & $7,692 \%$ \\
\hline
\end{tabular}

A tabela 4 deve ser complementada com a informação de que Toledo e Szafir-Goldstein são co-autores de três artigos. Isso significa que os três pesquisadores nacionais mais produtivos no período de tempo considerado e nos dois eventos analisados produziram, quando somados, um total de seis artigos.

A análise das citações dos artigos que integraram a amostra permitiu elaborar as tabelas 5 e 7, que apresentam, respectivamente, os autores nacionais e estrangeiros que receberam o maior número de citações. As tabelas 5 e 7 apresentam de forma consolidada as citações recebidas pelos

Revista Ibero-Americana de Estratégia - RIAE, São Paulo, v. 10, n. 1, p. 55-76, jan/abr. 2011. 
João Paulo Lara de Siqueira, Daniel Portillo Serrano, Irene Pereira de Oliveira Stenzel Rimonato \& Regina Tartareli.

11 autores apontados inicialmente pelos especialistas como prováveis fontes de citação e que obtiveram mais de uma citação e também dos autores que, posteriormente, durante a análise dos artigos, apresentaram mais de uma citação. Autores que foram citados apenas uma vez não constam nas tabelas 5 e 7 . Nessas tabelas também são apresentados os impactos das citações dos autores, calculados conforme indicado na metodologia.

Tabela 5 - Autores nacionais mais citados.

\begin{tabular}{|l|c|c|}
\hline \multicolumn{1}{|c|}{ AUTOR } & $\begin{array}{c}\text { TOTAL DE } \\
\text { CITAÇÕES }\end{array}$ & IMPACTO \\
\hline CASAROTTO FILHO, NELSON & 7 & $26,923 \%$ \\
\hline PIRES, LUIS HENRIQUE & 7 & $26,923 \%$ \\
\hline AMATO NETO, JOÃO & 5 & $19,231 \%$ \\
\hline ZACCARELLI, SERGIO B. & 4 & $15,385 \%$ \\
\hline TOLEDO, GERALDO L. & 5 & $19,231 \%$ \\
\hline SUZIGAN, WILSON & 3 & $11,538 \%$ \\
\hline SZAFIR-GOLDSTEIN, CLÁUDIA & 3 & $11,538 \%$ \\
\hline LASTRES, HELENA M. M. & 2 & $7,692 \%$ \\
\hline
\end{tabular}

A tabela 6 apresenta as áreas de atuação dos autores nacionais mais citados e as instituições às quais eles estão ligados.

Tabela 6 - Áreas e instituições dos autores nacionais mais citados.

\begin{tabular}{|l|c|c|}
\hline \multicolumn{1}{|c|}{ AUTOR } & ÁREA & INSTITUIÇÃo \\
\hline AMATO NETO, JOÃO & Produção & USP \\
\hline CASAROTTO FILHO, NELSON & Produção & UFSC \\
\hline LASTRES, HELENA M. M. & Economia & UFRJ \\
\hline PIRES, LUIS HENRIQUE & Não disponível & Não disponível \\
\hline SUZIGAN, WILSON & Economia & UNICAMP \\
\hline SZAFIR-GOLDSTEIN, CLÁUDIA & Administração & MACKENZIE \\
\hline TOLEDO, GERALDO L. & Administração & USP \\
\hline ZACCARELLI, SERGIO B. & Administração & USP/UNIP \\
\hline
\end{tabular}

Tabela 7- Autores estrangeiros mais citados.

\begin{tabular}{|l|c|c|}
\hline \multicolumn{1}{|c|}{ AUTOR } & $\begin{array}{c}\text { TOTAL DE } \\
\text { CITAÇÕES }\end{array}$ & IMPACTO \\
\hline PORTER, MICHAELE. & 25 & $96,154 \%$ \\
\hline MARSHALL, ALFRED & 6 & $23,077 \%$ \\
\hline MEYER-STAMER, JÖRG & 5 & $19,231 \%$ \\
\hline KRUGMAN, PAUL & 4 & $15,385 \%$ \\
\hline SCHIMITZ, HUBERT & 4 & $15,385 \%$ \\
\hline VALDÉS, JÉSUS A. & 4 & $15,385 \%$ \\
\hline ROSENFELD, STUART A. & 3 & $11,538 \%$ \\
\hline ALTENBURG, TILMAN & 2 & $7,692 \%$ \\
\hline CASTELLS, MANUEL & 2 & $7,692 \%$ \\
\hline
\end{tabular}

Revista Ibero-Americana de Estratégia - RIAE, São Paulo, v. 10, n. 1, p. 55-76, jan/abr. 2011. 
Uma Avaliação da Produção Acadêmica Brasileira Recente Sobre Clusters de Negócios.

Dentro do intervalo de tempo considerado nesta investigação, os anos de 2004 a 2006 foi o período em que houve maior produção nos eventos Enanpad e Semead sobre o tema clusters de negócios. Os 26 artigos apresentados nesses eventos, nas áreas de estratégia e administração geral, foram escritos por 62 autores, resultando em uma média de 2,4 autores por artigo. Com relação a esse conjunto de autores, verificou-se que a grande maioria deles (59 autores) apresentou apenas um trabalho. Três autores, Toledo, Szafir-Goldstein e Câmara, apresentaram mais trabalhos, nas quantidades de 4, 3 e 2, respectivamente. Esses três pesquisadores são, portanto, os mais produtivos, podendo-se acrescentar que os dois primeiros são ligados à área de administração e a terceira, à da economia.

Dos autores da tabela 7, certamente não causa espanto a presença em primeiro lugar de Michael Porter, autor da obra mais conhecida sobre clusters, o livro Vantagem Competitiva das Nações, que extrapolou os limites das leituras acadêmicas. Os economistas Marshall e Krugman também são autores que, por razões diversas são bastante conhecidos. Marshall por sua importância histórica na Economia e Krugman por ser um dos mais atuantes da atualidade, tendo recebido um prêmio Nobel.

Altenburg, Schimitz e Meyer-Stamer publicaram artigos na edição de 1999 do periódico World Development (volume 27, número 7), totalmente dedicado ao tema clusters. Castells, embora sendo de outra área, a sociologia, é também um autor conhecido além dos limites acadêmicos. Rosenfeld, por sua vez, é autor de um artigo (Bringing Business Clusters into the Mainstream of Economic Development, publicado no periódico European Planning Studies, em 1997) considerado pelos especialistas como sendo bastante importante.

\section{CONSIDERAÇÕES FINAIS}

As informações obtidas pela análise dos artigos apresentados nos eventos mencionados e no período de tempo considerado permitiu, dentro dos limites do método empregado, atingir os objetivos do estudo.

O primeiro objetivo, que era a identificação dos autores nacionais mais produtivos consta da tabela 4, que relaciona os autores que mais trabalhos apresentaram. Toledo, Szafir-Goldstein e Câmara são os pesquisadores mais produtivos.

Identificar os autores nacionais mais citados por autores nacionais (isto é, que têm maior impacto sobre aqueles que pesquisam clusters), era o segundo objetivo específico. Os 
pesquisadores nacionais que receberam maior número de citações por outros autores nacionais são os que estão listados na tabela 5. Como já mencionado, foi observado que esses autores são também autores de livros sobre o tema. O terceiro objetivo, semelhante ao segundo, mas relativo aos autores internacionais mais citados, levou à elaboração da tabela 7.

O quarto objetivo do presente estudo era, com base na lei de Lotka, conforme apresentada por Price, verificar a relevância dos autores nacionais na produção sobre clusters de negócios. Segundo essa proposição, seria esperado que $1 / 3$ da literatura fosse produzida por menos de 1/10 dos autores mais produtivos. Como o total de autores foi 62 e apenas três podem ser considerados os mais produtivos, 1/10 dos mais produtivos corresponderia a apenas um autor e 1/3 da literatura a, aproximadamente, nove artigos. Como o pesquisador mais produtivo, Toledo, é autor de quatro textos e não nove, não é possível dizer que a literatura analisada segue os padrões previstos por Lotka e Price.

De forma geral, observou-se que há predominância da área de administração em relação aos autores mais produtivos em clusters de negócios e também em relação aos autores nacionais mais citados (neste caso específico, em situação de igualdade com a área de produção), ao passo que entre as citações internacionais, com exceção de Porter, praticamente todas as demais citações são da economia. O fato de os padrões de Lotka/Price não terem sido observados sugere que a produção nacional de literatura sobre clusters encontra-se mais dispersa do que a de outras áreas do conhecimento.

Espera-se que o presente estudo tenha contribuído para o mapeamento da produção brasileira sobre clusters de negócios e que isso auxilie os pesquisadores da área a selecionar textos e autores para embasar seus trabalhos. O fato de haver uma razoável dispersão da produção sobre clusters sugere que no Brasil há a possibilidade de que pesquisadores venham a se consolidar como especialistas nessa área. Do ponto de vista da bibliometria, este estudo aponta as características da produção sobre clusters, de forma semelhante ao que, por exemplo, Urbizagástegui Alvarado (2002) fez com outras áreas do conhecimento.

É importante frisar que devido ao tamanho reduzido da amostra - 26 textos - a utilização de técnicas estatísticas mais sofisticadas ficou prejudicada e que todas as conclusões valem apenas para o conjunto de artigos e autores analisados. Por conta disso, imagina-se como desenvolvimento posterior deste estudo a pesquisa do mesmo assunto nos periódicos com os melhores conceitos da CAPES, assim como uma análise das citações dos textos internacionais mais representativos. A análise da rede formada pelas citações poderia ser feita com o auxílio de softwares como o UCINET 6.0 . 
Uma Avaliação da Produção Acadêmica Brasileira Recente Sobre Clusters de Negócios.

\section{REFERÊNCIAS}

Acedo, F. J.; Barroso, C.; Galan, J. L. The reource-based theory: dissemination and main trends. Strategic Management Journal, Vol. 27, 2006, pp. 621-636.

http://dx.doi.org/10.1002/smj.532

Altenburg, T.; Meyer-STamer, J. How to promote clusters: policy experiences from Latin America. World Development. V. 27. N.9, 1999, pp. 1963-1713.

http://dx.doi.org/10.1016/S0305-750X(99)00081-9

Araújo, C. A. Bibliometria: evolução histórica e questões atuais. Em Questão, Vol. 12, N. 1, Jan/Jun, 2006, Porto Alegre, pp. 11-32.

Balestrin, A.; Verschoore, J. R.; Reyes JR, E. O campo de estudo sobre redes de cooperação interorganizacional no Brasil. RAC, V. 14, N. 3, 2010, pp. 58-477.

Benetti, K. C.; Almeida, M. I. R.; Pereira, M. F.; Melo, P. A.; Roczanski, C. R. M. O Estado da Arte em Estratégia na Revista de Administração Contemporânea: um estudo bibliométrico. Anais do XI Semead. São Paulo, 2008, pp. 1-16.

Bergstrom, C. T.; West, J. D. Assessing citations with the Eigenfactor TM Metrics. Neurology, V. 71, 2008, pp.1850-1851.

http://dx.doi.org/10.1212/01.wnl.0000338904.37585.66

Dombrow, J.; Turbull, K. T. Individuals and institutions publishing in research in real state 19891998. Journal of Real Estate Literature; Vol. 10, N. 1, 2002, pp. 45- 91.

Boyd JR, H. W.; Westfall, R. Pesquisa mercadológica: textos e casos. Rio de Janeiro: FGV, 1979.

Guedes, V. L. S.; Borschiver, S. Bibliometria: uma ferramenta estatística para a gestão da informação e do conhecimento, em sistemas de informação, de comunicação e de avaliação científica e tecnológica. Anais do Encontro Nacional de Ciências da Informação, Salvador/BA, 2005. Disponível em: www.cinform.ufba.br/vi_anais/docs/VaniaLSGuedes.pdf. Acessado em 09/07/2009.

Journal Citation Reports: disponível em http://thomsonreuters.com/products_services/science/academic/impact_factor/ acessado em 18/04/2011 às 20:00.

Krugman, P. Geography and trade. Cambridge: MIT Press, 1991.

Lastres, H.M.M.; Cassiolato, J.E. Glossário de arranjos e sistemas produtivos e inovativos locais. Rio de Janeiro: UFRJ / Instituto de Economia. Nov. 2003. Disponível em www.redesist.ie.ufrj.br . Acessado em 26/02/2009.

Revista Ibero-Americana de Estratégia - RIAE, São Paulo, v. 10, n. 1, p. 55-76, jan/abr. 2011. 
Lima, R. C. M. Estudo bibliométrico: análise de citações no periódico "scientometrics". Ci. Inf., V. 13, N. 1, 1984, pp. 57-66.

Lunardi, G. L.; Rios, L. R.; Maçada, A. C. F. Pesquisa em Sistemas de Informação: uma análise a partir dos artigos publicados no Enanpad e nas principais revistas nacionais de Administração. Anais do XXIX Enanpad, 2005.

Marconi, M. A.; Lakatos, E. M. Fundamentos de metodologia científica. 6. ed. São Paulo: Atlas, 2009.

Marshall, A. Princípios de Economia. V. 1. São Paulo: Abril Cultural, 1982.

Moretti, S. L. A., Campanário, M. A. A Produção intelectual brasileira em responsabilidade social empresarial - RSE sob a ótica da bibliometria. RAC, V. 13, Edição Especial, 2009, pp. 68-86.

Oliver, A. L.; Ebers, M. Networking network studies: an analysis of conceptual configurations in the study of inter-organizational relationships. Organization Studies, V. 19, N. 4, 1998, pp. 549-583.

http://dx.doi.org/10.1177/017084069801900402

Paula, A. P. P.; Maranhão, C. M. S. A.; Barreto, R. O.; Klechen, C. F. A tradição e a autonomia dos estudos organizacionais críticos no Brasil. RAE, V. 50, N.1, 2010, pp. 10-23.

Pegino, P. M. F. As bases filosóficas das publicações na área de estratégia das organizações nos encontros nacionais da Anpad. Anais do XXIX Enanpad, 2005.

Perry, M. Business clusters: an international perspective. New York: Routledge, 2005. http://dx.doi.org/10.4324/9780203310694

Porter, M. E. Clusters and the new economics of competition. Harvard Business Review, 1998, pp. 77-90.

Porter, M. E.. The Competitive Advantage of Nations. New York: The Free Press, 1990.

Pritchard, A. Statistical bibliography or bibliometrics? Journal of Documentation, V. 25, N.4, 1969, pp. 348-349.

Rosenfeld, S. A. Bringing Business Clusters into the Mainstream of Economic Development. European Planning Studies, V. 5, N. 1, 1997, pp. 03-23.

Strehl, L. O fator de impacto do ISI e a avaliação da produção científica: aspectos conceituais e metodológicos. Ci. Inf., V. 34, N. 1, 2005, pp.19-27.

http://dx.doi.org/10.1590/S0100-19652005000100003

Revista Ibero-Americana de Estratégia - RIAE, São Paulo, v. 10, n. 1, p. 55-76, jan/abr. 2011. 
Uma Avaliação da Produção Acadêmica Brasileira Recente Sobre Clusters de Negócios.

Suzigan, W.; Furtado, J.; Garcia, R.; Sampaio, S.E.K. Sistemas locais de produção: mapeamento, tipologia e sugestões de políticas. Anais do XXXI Encontro Nacional de Economia. Porto Seguro, Dezembro, 2003.

Urbizagástegui Alvarado, R. A Lei de Lotka na bibliometria brasileira. Ci. Inf., V. 31, N. 2, 2002, pp. 14-20.

Van Leeuwen, T. The application of bibliometric analyses in the evaluation of social science research. Who benefits from it, and why it is still feasible. Scientometrics, V. 66, N. 1, 2006, pp. 133-154.

Zaccarelli, S. B. Estratégia e sucesso nas empresas. São Paulo: Saraiva, 2004.

Zaccarelli, S. B.; Telles, R.; Siqueira, J.P.L.; Boaventura, J.M.G.; Donaire, D. Clusters e redes de negócios: uma nova visão para a gestão dos negócios. São Paulo: Atlas, 2008.

Recebido: 08/12/2010

Aprovado: 16/02/2011

Revista Ibero-Americana de Estratégia - RIAE, São Paulo, v. 10, n. 1, p. 55-76, jan/abr. 2011. 